\title{
Die Erwärmung der Wohnungen durch die Sonne.
}

Von

Prof. E. v. Esmarch

in Göttingen.

Die Wohnungshygiene hat in den letzten Jahrzehnten in wissenschaftlicher wie praktischer Beziehung zweifellos grosse Fortschritte gemacht. Vor allen Dingen lassen sich diese auf dem Gebiete der künstlichen Erleuchtung und Erwärmung erkennen. Demgegenüber sind andere Fragen, die ebenfalls mit dem gesunden und behaglichen Bewohnen unserer Häuser in innigem Zusammenhang stehen, merkwürdiger Weise noch gar nicht oder kaum berücksichtigt worden, dazu gehört unter anderem die künstliche Kühlhaltung unserer Zimmer.

Dass eine Ueberwärmung der letzteren, namentlich in der heissen Jahreszeit bei uns nicht selten vorkommt, wird von keinem geleugnet werden, auch die schädlichen, und zum wenigsten unangenehmen Wirkungen derselben sind allgemein bekannt, weil sie eben allgemein empfunden werden, dazu gehören z. B. alle die Erscheinungen, wie Unlust oder Unfähigkeit zur Arbeit, Schlaflosigkeit u. s. w., die sich bei Aufenthalt in zu warmen Räumen bald einzustellen pflegen, dazu ferner aber auch indirecte Schädigungen, wie das Verderben unserer Nahrungsmittel, die in ihrer Wirkung dem Laien zwar weniger zum Bewusstsein kommen, dem Hygieniker aber sehr wohl bekannt sind; müssen wir doch z. B. die jedes Jahr im Sommer rapide in die Höhe schnellenden Zahlen der Todesfälle der Säuglinge mit in erster Linie auf diesen Grund zurückführen.

Es ist also ganz gewiss Grund genug vorhanden, dass wir uns etwas mehr, wie bisher geschehen, mit der Ueberwärmung unserer Häuser und deren Kühlhaltung beschäftigen. Soweit letztere künstlich geschehen 
kann, sind ja auch schon einige Maassregeln zu verzeichnen. Unsere Schlachthöfe und Markthallen sind vielfach mit solchen Kühlräumen versehen und auch für grössere öffentliche Gebäude, Theater und Versammlungssäle hat man schon hier und da Anfänge gemacht, die die Technilker wohl zum weiteren Fortschreiten auf diesem Gebiet anspornen könnten.

Im Grossen und Ganzen sind es aber bisher nur Anfänge gewesen und es ist nach Allem wohl keine grosse Hoffnung rorhanden, dass wir die künstliche Kühlung auch für unsere Privaträume in ausgedehntem Maasse in nächster Zeit werden heranziehen können, dazu werden die Einrichtungen eben leider wohl zu theuer sein und auch wohl bleiben.

Aber vielfach wird eine künstliche Kühlung auch gar nicht nöthig sein, wenn wir dem Uebel einen Schritt weiter entgegengehen und von vorneherein eine Ueberwärmung zu verhindern suchen, also nicht therapeutisch sondern prophylaktisch verfahren.

Fragen wir uns nun zunächst einmal, wodurch werden denn unsere Rāume überwärmt, so lautet für die warme Jahreszeit wenigstens die Antwort, in erster Linie durch die Sonne, ihr gegenüber treten im Sommer die anderen Wärmefactoren, wie Kochherde, die Menschen selbst mit ihrer Wärmeausstrahlung u. s. w. meist ganz in den Hintergrund.

Das ist ja auch lange bekannt und die Praxis hat daraus auch schon ihre Consequenzen gezogen; so werden wenn möglich die Rüume zur Aufbewahrung von Lebensmitteln in unseren Wohnungen nach Norden verlegt, durch Isolirschichten, dicke Mauern, schlecht die Wärme leitendes Baumaterial, durch weissen Anstrich der Wände oder in südlichen Lündern auch wohl durch Verkürzung der Strassenbreiten sucht man rationell die Sonnenwirkung abzuschwächen, ebenso werden Markisen, Jalousieen und ähnliche Vorrichtungen vor unseren Fenstern nicht allein zum Abhalten ron Licht sondern auch von Wärme angewendet. Auch wissenschaftlich sind nach dieser Richtung hin schon Untersuchungen angestellt, so kennt man genauer die Wärmeaufnahme durch die Sonne bei verschiedenen Farben, unsere üblichen Baumaterialien sind in ihrer verschiedenen Wärmeleitungsfähigkeit auch ziemlich bekannt, soviel ich aber weiss, sind directe Versuche, wie sich die bei uns zum Schutze gegen die Sonne gebräuchlichen Nittel gegen einander verhalten, noch nicht weiter angestellt worden.

So möchte ich glauben, dass einige Untersuchungen, die diese Frage berühren und zu denen mir der diesjährige sonnenreiche Sommer besonders hülfreich gewesen ist, soweit interessiren, dass ich sie hiermit der Oeffentlichkeit übergeben darf.

Allerdings möchte ich vorweg bemerken, wie das übrigens jeder wohl von vorneherein einsehen wird, dass meine Versuche nicht ohne Weiteres mit den Einrichtungen unserer Häuser verglichen oder ihnen gleichgestellt 
werden dürfen, aber ich denke, dass sie doch nach mancher Richtung hin. ganz wichtige Fingerzeige auch für die Praxis ergeben werden.

Ich lasse zunächst eine kurze Beschreibung meiner Versuchsanordnung folgen. Ich liess mir zwei ganz gleiche Holzlästen bauen, deren innere lichte NIaasse je $46^{\mathrm{cm}}$ Länge, $25^{\mathrm{cm}}$ Breite und $27.5^{\mathrm{cm}}$ Tiefe betrugen. Das Holz war $2^{\mathrm{cm}}$ stark und wurde allseitig mit $5^{\mathrm{cm}}$ starken Korkstein dicht umschlossen, welcher aussen mit weissem Oelfarbenanstrich versehen wurde. Auf diese Weise wurden die Kästen vor Wärmetransmission von aussen wie von innen derartig geschützt, dass sie, für meine Versuche wenigstens, einwandsfreie vergleichbare Resultate geben mussten. Die Deckel der Kästen waren abnehmbar und wurden bei den Versuchen durch die zu prüfenden Abdeckungen und Wärmeschutzvorrichtungen ersetzt, die genau nach den Kästen zugeschnitten waren und zum Ueberfluss, wenn nöthig noch mit Watte an ihrem in den Korkstein eingreifenden Rande gedichtet wurden.

Vor Beginn der Versuche wurle in jeden Kasten ein Thermograph hineingestellt, deren guter gleichmässiger Gang durch besondere Versuche vorher festgestellt war, dann wurden die Kästen im Innern eines grossen Saales je morgens bis 11 Uhr vor ein Ost-, sodann vor ein Südfenster gesetzt mit einer Neigung der oberen Oeffnung zum Fenster von $10^{\circ}$, nur in den Jalousieversuchen 24 und 25 betrug die Neigung $45^{\circ}$. Auf diese Weise wurden beide Kästen immer möglichst intensiv und durchaus gleichmässig von der Sonne bestrahlt. Ton einer Aufstellung im Freien wurde Abstand genommen, um die etwaige Wirkung ungleich abkühlender Luftströmungen, die ron aussen die Kästen hätten treffen können, zu vermeiden. Die Kästen wurden darauf mit den zu prüfenden Materialien geschlossen und daneben ein Maurer'scher Sonnenscheinmesser aufgestellt, der selbst wenige Minuten dauernde Wolkenschatten noch sehr gut erkennen liess. Als Sonnenscheindauer ist stets die gesammte an einem Versuchstage auf die Kästen fallende Sonnenzeit berechnet, und sind dabei also die während des Versuchs vorkommenden Wolkenschattenminuten abgezogen, wie bei den Versuchen unter Bemerkungen (Tabelle I) noch weiter ersichtlich ist.

Es ist klar, dass dadurch die einzelnen. Versuche nicht ohne Weiteres genau vergleichbar sind; denn eine beispielsweise 2 stündige ununterbrochene klare Sonnenbestrahlung wird roraussichtlich anders wirken, als eine ebensolange Bestrahlung, die sich durch Wolken vielfach unterbrochen, vielleicht auf einen ganzen Nachmittag ausdehnt, und ebenso grosse Fehler werden wohl durch gelegentliche dünne Verschleierungen des Himmels, durch verschiedenen Hochstand der Sonne - die Versuche erstreckten sich ron Mai bis September - und anderes anzunehmen 
sein, sodass genau genommen immer nur die beiden Versuche, die zugleich ausgeführt wurden, verglichen werden könmen.

So zeigen z. B. die Versuche 15, 16 und 17, die mit dem gleichen Deckmaterial vorgenommen wurden, erhebliche Unterschiede, wenn man sie, wie in Tabelle II geschehen, nach einzelnen Sonnenstunden rangirt, indem in $17 \mathrm{~b}$ wach 9 Stunden ununterbrochener Sonne pro Stunde die Erwärmung nur $0.88^{\circ}$, in $16 \mathrm{~b}$ dagegen, mit gleicher Expositionszeit aber im Ganzen nur 1 Stunde Sonne die stündliche Erwärmung auf $3.5^{\circ} \mathrm{zu}$ berechnen war. Hier wird wohl angenommen werden dürfen, dass die Haupterwärmung nicht durch die Sonne, sondern durch die umgebende Zimmerluft, in der die Kästen so lange standen, erfolgt ist. Das geht wenigstens mit einiger Wahrscheinlichkeit aus Versuch 8 herror, der gleichsam als Controle ganz ohne Sonne vorgenommen wurde; hier betrug nach 8 Stunden die Temperatur in beiden Kästen $2^{\circ}$ mehr, was sich nur durch die Uebertragung der höheren Zimmertemperatur auf die Kästen erklären lässt und eine Stütze findet in den ganz langsam und gleichmässig ansteigenden Temperaturcurven in diesem Fall.

Versuch 7 a zeigt andere Sonnenstundenwerthe wie $11 \mathrm{~b}$, obgleich das Deckmaterial und die Zabl der Sonnenstunden gleich war, aber in $11 \mathrm{~b}$ war die Sonne vielfach durch Wolken verdeckt, während sie in $7 \mathrm{a}$ ununterbrochen schien, was nicht ohne Einfluss bleiben konnte, wie übrigens auch aus dem verschiedenen Gang der beiden Temperaturcurven deutlich hervorging, die in 7 a ganz gleichmässig anstieg, in $11 \mathrm{~b}$ mehrfache Depressionen zeigte.

So giebt denn die Tabelle II, die ich in der Weise hergestellt habe, dass ich die Gesammterwärmung der einzelnen Versuche in die Gesammtanzahl der registrirten Sonnenstunden dividjrte und nach den so ermittelten Werthen die Reihenfolge bildete, nicht genall den Schutz in absteigender Linie wieder, den die einzelnen Deckmaterialien gegen die Sonnenerwärmung gewähren, im Allgemeinen aber wird, denke ich, die Tabelle doch lehrreich und für die Praxis brauchbar sein.

Die Tabelle I enthält die Versuche in der Reihenfolge, wie sie vorgenommen wurden, und wird ohne Weiteres verständlich sein, so dass ich nunmehr noch zu einer kurzen Besprechung in Einzelnen übergehen kann.

Bei der Erwärmung unserer Wohnungen durch die Sonne spielt, wie schon oben kurz erwähnt, die verschiedene Wärmeleitungsfähigkeit der schützenden Wände, Dächer u.s. w. eine grosse Rolle und es lag nahe, wenn auch die Leitungsfähigkeit der gebräuchlichen Materialien bereits ziemlich gut bekannt ist, nach dieser Richtung hin einige Versuche mit Baumaterial, wie es zumeist in der Praxis gebraucht wird, anzustellen. So wurden denn aus diesem Grunde zunächst die verschiedenen bei uns 
gebräuchlichen Dachabdeckungen, wie Pfannen, Schiefer, Pappe, Zinkblech mit und ohne Holzverschalung oder anderen isolirenden Unterlagen mit einander zu vergleichen versucht. Man beachte vor Allem die Versuche $9,12,19,20,36,39,43.9$ und 12, wenn auch an verschiedenen Tagen angestellt, lassen sich wohl direct mit einander vergleichen, da an beiden Tagen die ganze Zeit hindurch, bei 12 nur mit einer kurzen Unterbrechung, intensiver Sonnenschein herrschte. Der Versuch entscheidet hier zu Gunsten des Schiefers, d. h. Schieferdach schützt also mehr wie Dachpappe, und ebenso auch mehr wie Zinkblech (19), während umgekehrt Schiefer wieder mehr Wärme durchlässt, wie Pfannendach (20 und 36). Vor Allem deutlich aber ist überall der grosse Schutz, den unter dem Dachmaterial angebrachte Isolirschichten bewirken, wie eine gewöhnliche Holzverschalung. Das zeigen z. B. 9, 12 und 39. In 9 beträgt der dadurch erzielte Wärmeschutz etwa 50 Procent, in 12 beinahe 100 Procent und in 43 wird Dachpappe durch Holzverschalnng dem gewöhnlichen Pfannendach im Wärmeschutz fast gleich.

Nächst der Wärmeleitungsfähigkeit kommt, wie ebenfalls schon ausgeführt, die Farbe der der Sonne zugekehrten Oberfäche in Frage und wie gross der Einfluss dieses Factors ist, ist aus den Versuchen 1, 2, 5, 7, 15 bis 18, 24 bis 27, 30 und 41, 42 zu erkennen. Der gleiche Holzbelag schwarz gestrichen (1) berirkt schon nach 2 Stunden Sonnenwirkung die doppelte Erwärmung, wie ein solcher mit weissem Anstrich und noch deutlicher tritt der Einfluss der Farbe bei 2 hervor, wo an Stelle des Holzes dünne Pappe genommen worden war. Auch Versuch 42 zeigt recht anschaulich, wie durch Ankalken einer Pappdachabdeckung ein erheblicher Wärmeschutz erzielt wird.

Umgekehrt wird durch ein Schwärzen der rothen Dachziegel, wie solches vielerorts gebräuchlich ist, die Wärmeaufnahme bei Sonnenschein erhöht (Versuch 7) und ist daher dieses Verfahren, welches auch aus ästhetischen Gründen keine Empfehlung verdient, da es zumeist dem Hause ein düsteres unfreundliches Ansehen zu geben pflegt, ebenso von Seiten der Hygiene zn verwerfen. Die Technik sollte daher andere Mittel anwenden, als das Schwārzen, wenn es wirklich nöthig ist, ein Ziegeldach gegen Witterungseinflüsse haltbarer zu machen.

Selbstrerständlich tritt der Einfluss der Farbe um so mehr zurück, je dicker und weniger gut wärmeleitend im Uebrigen die schützenden Abdeckungen sind; dafür sind gute Beispiele Versuch 15 bis 17, 18 und ebenso $24,27,30$ und 41 , auf welch' letztere ich nachher noch zurückkommen muss, aber es wäre doch falsch hieraus zu schliessen, dass bei guter Isolirung die Farbe der obersten Schicht gleichgültig ist für die Erwärmung der darunter befindlichen Räume. Denn es ist zu berück- 
sichtigen, dass die isolirenden Schichten die Wärme auch länger zu halten rermögen, und schliesslich nach tage- oder wochenlanger Besonnung doch erheblich mehr Wärme nach innen transmittirt wird, wenn eben von aussen durch die dunkle Farbe mehr aufgenommen wird. Um dieses zahlenmässig zu beweisen, reichen natürlich meine Versuche, die sich immer nur auf einen Tag ausdehnen liessen, nicht aus. Man wird daher auch fernerhin gut thun, Räume, welche man besonders gegen Sonnenerwärmung schützen will, mit einem hellen Anstrich zu rersehen, selbst wenn darunter noch dicke Isolirschichten vorhanden sind.

Eins der wesentlichsten Mittel zur Wärmeregulation unserer Wohnungen, sowohl im Winter wie im Sommer, besitzen wir in den Fenstern. Selbst in geschlossenem aber sonst nicht besonders geschützten Zustande verlieren wir eine erhebliche Menge von Wärme dadurch im Winter und empfangen umgekebrt solche im Sommer, vor Allem bei Sonnnenbestrahlung. Wie sehr hoch dieser letztere Factor sein kaun, illustriren so recht die Versuche 13, 14 und 21. Zugleich zeigen sie uns aber, welchen Schutz schon ein einfacher Leinenrorhang gegen diese Erwärmung geben kann. Bedeutend weniger gut schützt dagegen ein Store $(28,40)$, durch dessen grosse Maschen eben doch eine Menge Sonnenstrahlen noch ungehindert durchpassiren.

Bei den anderen üblichen Fenstervorhäugen, von denen eine ganze Reihe zur Vergleichung herangezogen wurden, (siehe 18, 22 bis 30,40 und 41), ist von Ausschlag einmal die Furbe und sodann die Dicke des Stoffes. So überwiegt z. B. in 26 der letztere Factor, wenn auch wenig, da unter dem dickeren graugelblichen Leinenstoff die Erwärmung geringer war, wie unter dem weissen Leinen. Nichtsdestoweniger wird man für die Praxis, so in Schulen, dem letzteren Stoff zu Vorbängen den Vorzug geben, da der erhöhte Wärmeschutz in diesem Fall wohl mehr wie aufgehoben wird durch die starke Verdunklung, die durch das Vorziehen der Vorhänge im Zimmer bewirlst wird.

Interessant ist auch ein Vergleich von 18a und b, wo zwei Stoffe annähernd gleicher Dichtigkeit und Dicke, aber rerschiedener Farbe genommen waren, hier musste der Versuch zu Gunsten von weiss entscheiden. In 27 und 30 dagegen ist wiederum die Dicke des Vorhanges massgebend und die helle Gardine tritt in ihrer Schutzwirkung mehr oder weniger deutlich zurück; wird aber wie in 41 der helle Vorhang doppelt genommen, so hat er wieder 'selbst gegenüber einen dicken, doppelten, schwarzen Wollrorhang die Oberhand. Sehr rationell muss es natürlich auch erscheinen, wenn man einen dunklen Vorhang nach aussen mit einem helleren Ueberzug versieht (29) und man wird also für die Praxis sich die Regel merken dürfen, dass man als Sonnenschutz zu Vorhängen 
helle Stoffe, unter Umständen in doppelter Schicht oder wo es auf Verdunklung des Raumes weiter nicht ankommt, auch einmal einen dunklen Stoff, dann aber am besten mit einem hellen davor, nach aussen hin, zu nehmen hat.

Ausser den Vorhängen besitzen wir übrigens noch andere Schutzmaassregeln, die wir an oder vor unseren Fenstern gegen die Sonnenwärme anwenden können, z. B. die Doppelfenster. Dass diese nicht allein, wie genugsam bekannt, gegen Abkühlung im Winter, sondern auch im Sommer gegen Erwärmung recht nützlich sind, dürfte aus dem Versuch 22 gefolgert werden können, wenigstens, wenn man einen weissen Vorhang noch darüber zieht. Weiter kommen die Jalousien in Betracht, deren Natzen durch die Praxis, namentlich in den südlichen Ländern, sich tausendfältig bewährt hat, und die auch bei uns meiner Ansicht nach, mehr angewendet werden müssten, als es bisher geschehen ist. In der That scheint nach meinen Versuchen ihr Sonnenschutz ein ganz herrorragender zu sein, so in 25 und 24 , wo er doppelt so gross, in 23, wo er sogar drei Mal so gross war, wie der durch einen weissen Leinenvorhang gewährten. Zu beachten ist auch, dass es anscheinend zweckmässig ist, die Jalousie nicht unmittelbar vor dem Fensterglas anzubringen, sondern besser, wie es übrigens ja auch vielfach geschieht, einen Abstand zwischen Jalousie und Glas zu lassen (Versuch 23). Die Erklärung für den ganz besonderen Wärmeschutz in diesem Falle wird wohl darin zu finden sein, dass die Luft zwischen Jalousie und F'enster hier frei circuliren konnte, wodurch permanent eine grosse Menge Wärme abgeführt werden musste.

Der äussere Anstrich dagegen scheint von keinem besonderen Einfluss zu sein (vgl. 24 und 25) und tritt jedenfalls hinter die schützende Wirkung der schlechten Wärmeleitung und Luftcirculation so zurück, dass wir auch fernerhin getrost den wohl ziemlich allgemein gebräuchlichen grünen Anstrich unserer Jalousien als rationell empfehlen dürfen.

Sehr verbreitet namentlich für Veranden, Corridore, Closets aber auch manche andere Räume sind Verglasungen aus buntem oder anderem das Licht mehr oder weniger gut durchlassendem Glas und es wird oft angenommen, dass in solchen Fällen ein besonderer Schutz gegen Sonnenerwärmung nicht nöthig ist. Es lag nahe auch dieses experimentell etwas näher festzustellen, wie das in den Versuchen 21, 31 bis 35 und 38 geschehen ist. Zum Vergleich ist dabei meist ein weisser Vorhang hinter gewöhnlichem Fensterglas herangezogen und nur einige Male sind auch verschiedene Glassorten direct mit einander verglichen.

Das Ergebniss der Versuche ist kurz das, dass alle Glassorten mit Ausnahme ron Milchglas, welches etwas mehr, wie ein weisser Vorhang unter Fensterglas schützt (es war im Versuch 35 allerdings auch etwas 
dicker wie das Fensterglas $\left(3^{1} / 2: 2^{\mathrm{mm}}\right)$, sehr viel Sonnenwärme durchlassen, vor Allem Ornamentglas $(32,38)$, das dem gewöhnlichen klaren Glas kaum nachsteht (vgl. 13, 14 mit 32, 38), ebenso gewährt das gewöhnliche matte Glas sehr geringen Wärmeschutz (21), während bunte Gläser $(31,33)$ etwas mehr schützen, aber lange noch nicht so, wie ein weisser Vorhang hinter klarem Glas. Dasselbe gilt endlich von dickerem Rohglas, wie man es bäufig zu Verglasungen in Kellern u. s. w. verwendet. Es geht also aus dem Allen hervor, dass, wenn man wirklich Wārmeschutz bei Verglasung der verschiedenen Art haben will, man ohne Vorhänge nicht auskommen kann, was vor Allem für Schulen, Vorrathsräume aber auch sonst oft zu beachten sein wird.

Zum Schluss möchte ich die Aufmerksamkeit noch auf einen Wärmeschutz wenden, der, wenn auch vielfach angewendet und richtig gewürdigt, doch von vielen Hygienikern, Technikern und auch Laien verurtheilt und meiner Ansicht nach sehr zu Unrecht verurtheilt wird. Ich meine die Berankung unserer Häuser.

A priori, namentlich aber nach den oben angeführten Jalousieversuchen, ist wohl anzunehmen, dass der Schutz gegen Sonnenerwärmung durch Berankung ein recht beträchtlicher sein wird. Dieser Schutz liegt, ähnlich wie bei den Jalousieen hauptsächlich wohl in der beweglichen Luftschicht, die zwischen der sonnenbestrahlten Oberfläche und der Hauswand eingeschaltet wird, und so werden denn die Resultate meiner Versuche $(10,11,37)$ nicht gerade überraschen. Die letzteren wurden derart angestellt, dass Ranken von wildem Wein, frisch abgebrochen und mit dem unteren Ende in ein Gefäss mit Wasser gestellt, über die Oberfäche der Versuchskästen ausgebreitet wurden und zwar den natürlichen Verhältnissen möglichst entsprechend in 10 und 11 in so dicker Schicht, dass Sonnenstrahlen nirgends mehr direct auf die Kastenoberfläche gelangen konnten, in 37 dagegen so, dass noch an manchen Stellen die Sonne durchdrang, wie es bei sehr dünner Berankung ja auch gelegentlich wohl rorkommt.

Der Schutz dieser Berankung ist sehr deutlich selbst in letzterem Fall zu erkennen, in 10 und 11 war er so gross, dass die Temperatur im berankten Kasten nur halb so: hoch stieg, wie im Controlversuch.

So sprechen, glaube ich, die Versuche doch recht sehr zu Gunsten einer Berankung unserer Häuser, zumal die Gründe der Gegner sich in den meisten Fällen als nicht stichhaltig erweisen. Es wird zwar vielfach behauptet, dass eine Berankung die Hauswand leicht feucht mache; nach meiner Erfahrung ist eher das Gegentheil der Fall und es ist das eigentlich auch von vorneherein zu erwarten. Das Blätterdach gewährt gegen Niederschläge meist einen ganz ausgezeichneten Schutz und lässt solche 
überhaupt gar nicht bis an die Hauswand gelangen, die Feuchtigkeit aber, welche doch zwischen die Blätter eindringt, wird im Sommer wenigstens sehr schnell verdunsten, da der Luftzutritt ja ein ganz ungehinderter ist und die meist hohe Temperatur in der Blätterschicht diese Verdunstung noch befördert. Auch im Winter ist selbst in unserem feuchten Klima eine Durchnässung der Wand nicht zu befürchten, ich wenigstens wohne seit Jahren in einem Hause, welches an der Wetterseite theilweise mit dichtem Epheu überzogen ist, habe aber niemals an diesen Stellen etwas von Wandfeuchtigkeit bemerkt und möchte im Gegentheil glauben, dass diese Theile weniger von den atmosphärischen Niederschlägen zu leiden haben, wie die unbedeckten. Auch den häufig gehörten Einwand, dass durch Berankung des Hauses lästige Insecten leichter und reichlicher in's Haus kommen, kann ich aus eigener Erfahrung als nur selten zutreffend bezeichnen. Es ist ja richtig, dass wenn reife Trauben an der Wand hängen, sich dort auch Wespen reichlicher einfinden und gelegentlich in's Zimmer kommen können. Wer solches befürchtet, wird durch die Wahl anderer Rankgewächse, wilden Wein's z. B. diesen Uebelstand leicht zu vermeiden im Stande sein. Ich möchte daher noch einmal die Berankung rom Standpunkte des Hygienikers aus nachdrücklichst empfehlen und thue es schliesslich auch im Sinne des bekannten Prof. SchultzeNaumburg. So manches traurige Denkmal einer glücklicherweise jetzt absterbenden Stilperiode wird durch den milden Schleier eines Blätterdach's verhüllt und verbessert werden hönnen. 
E. v. Esmarch:

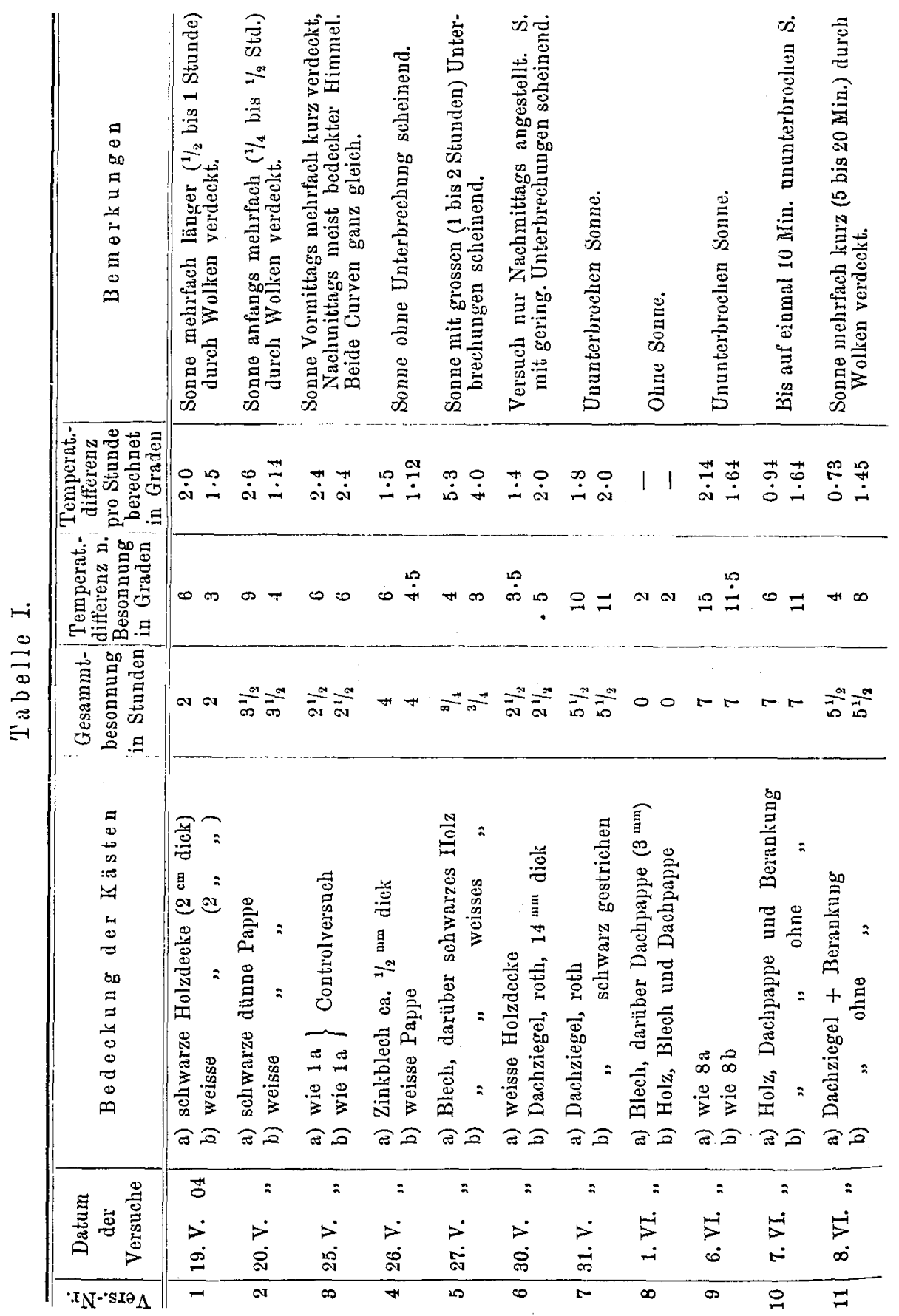


Die Erwïrmung Der Wohnungen durch de Sonne.

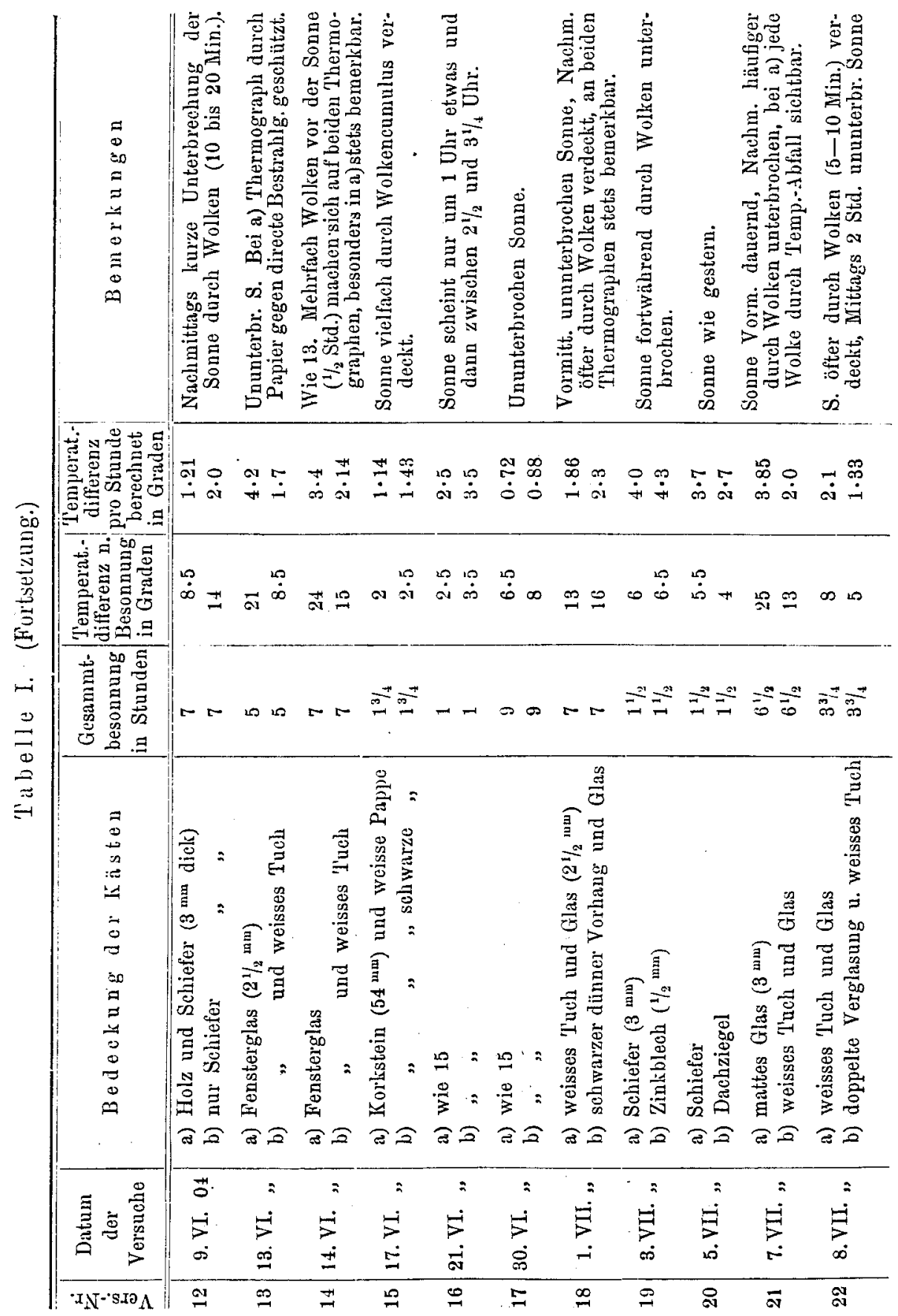


E. v. Esmarch:

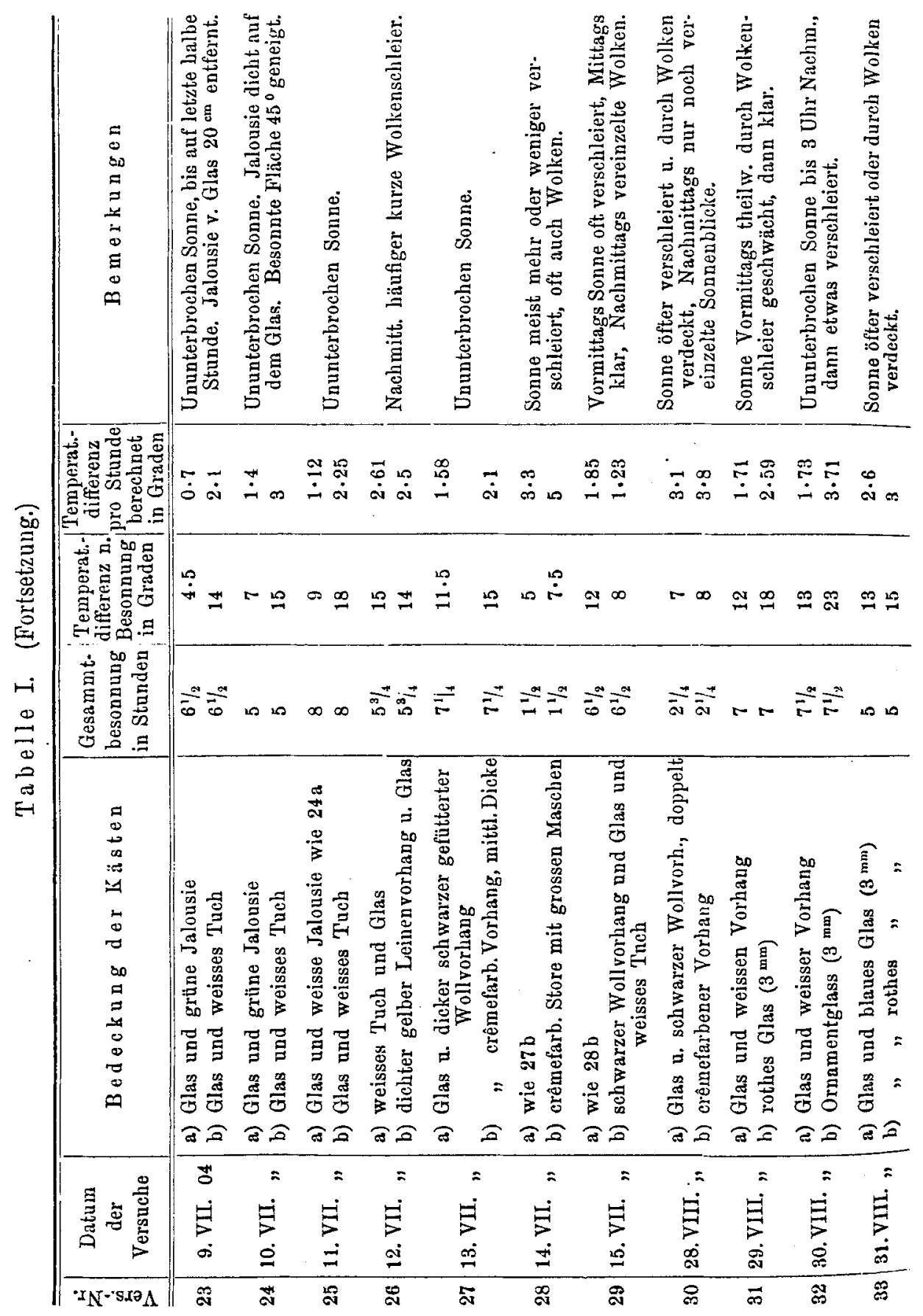


Die Erwärmung der Wohnungen dorch die Sonne.

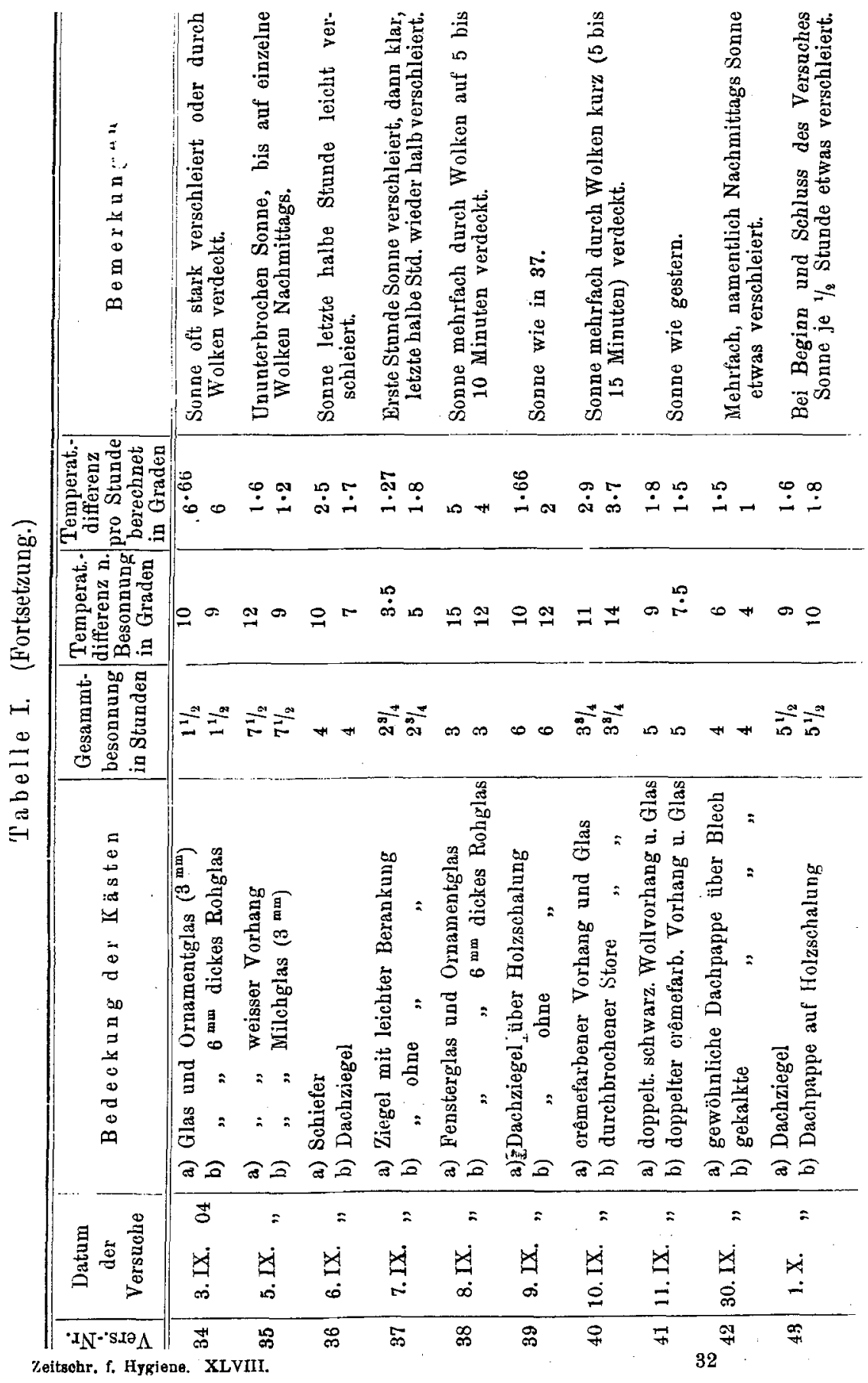


Tabelle II.

Stündliche Erwärmung durch Sonnenstrahlen bei verschiedener Bedeckung.

\begin{tabular}{|c|c|c|c|c|c|c|c|}
\hline 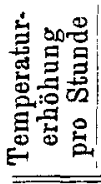 & $\begin{array}{c}z \\
z \\
\dot{1} \\
\dot{0} \\
\dot{0} \\
p\end{array}$ & 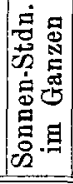 & Dec & 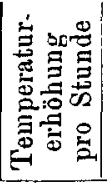 & 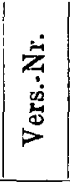 & 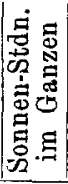 & De \\
\hline $0.7^{\circ}$ & $23 a$ & $6 \%$ & gräne Jalc & $2 \cdot 0$ & $7 \mathrm{~b}$ & $5 \%$ & Dachziegel scl \\
\hline $0.72^{\circ}$ & $17 \mathrm{a}$ & 9 & Korkstein mit weisser Pappe & $2 \cdot 0$ & $21 \mathrm{~b}$ & $61 / 2$ & weisses Tuch und Glas \\
\hline 0.73 & $11 \mathrm{a}$ & $5 \%$ & Dachziegel mit Berankung & $2 \cdot 0$ & $12 \mathrm{~b}$ & 7 & Schiefer \\
\hline 0.88 & $|17 \mathrm{~b}|$ & 9 & Korkstein m. schwarz. Pappe & $2 \cdot 1$ & $27 \mathrm{~b}$ & $71 / 4 \mid$ & crême Vorbang und Glas \\
\hline 0.94 & $10 \mathrm{a}$ & 7 & Holz a. Dachp. a. Ber & $2 \cdot 1$ & $22 \mathrm{a}$ & $3 \%$ & weisses Tuch und Glas \\
\hline $1 \cdot 0$ & $42 \mathrm{~b}$ & 4 & ss gekalkt & $2 \cdot 1$ & $23 \mathrm{~b}$ & $61 \frac{1}{2}$ & de \\
\hline $1 \cdot 12$ & $4 b$ & 4 & dünne $\mathrm{Pa}$ & $2 \cdot 14$ & $14 \mathrm{~b}$ & 7 & des \\
\hline $1 \cdot 12$ & $25 \mathrm{a}$ & 8 & weisse Jalon & $2 \cdot 14$ & $9 \mathrm{a}$ & 7 & Dachpappe \\
\hline $1 \cdot 14$ & $2 \mathrm{~b}$ & $31 / 2$ & weiss & 2.25 & $2 \bar{b}$ & 8 & weisses Tuch und Glas \\
\hline $1 \cdot 14$ & $15 \mathrm{a}$ & $19 / 4$ & Korkstein mit weisser Pappe & $2 \cdot$ & $18 \mathrm{~b}$ & 7 & schwarzer dünner Vorhang \\
\hline $1 \cdot 2$ & $35 \mathrm{~b}$ & $71 / 2$ & & & & & \\
\hline $1 \cdot 21$ & $12 \mathrm{a}$ & 7 & Holz & & $3 a$ & $21 / 2$ & Holz \\
\hline $1 \cdot 23$ & $29 \mathrm{~b}$ & $61 / 2$ & $\begin{array}{l}\text { weisses } \\
\text { Wolly }\end{array}$ & $\begin{array}{l}2 \cdot 5 \\
2 \cdot 5\end{array}$ & $\begin{array}{l}26 \mathrm{~b} \\
16 \mathrm{a}\end{array} \mid$ & $\begin{array}{l}5 \% / 4 \\
1\end{array}$ & $\begin{array}{l}\text { dicker gelber } \\
\text { Korkstein und }\end{array}$ \\
\hline $1 \cdot 27$ & $37 \mathrm{a}$ & $23 / 4$ & ter Berankg. & $2 \cdot 5$ & $36 \mathbf{a}$ & 4 & \\
\hline $1 \cdot 33$ & $22 \mathrm{~b}$ & $33^{3}$ & Tuch & $2 \cdot 59$ & $31 \mathrm{~b}$ & 7 & rothes Glas \\
\hline $1 \cdot 4$ & $24 \mathrm{a}$ & 5 & grüne Jalousie, dicht aufGlas & $2 \cdot 6$ & $2 \mathbf{a}$ & $31 / 2$ & schwarze \\
\hline $1 \cdot 4$ & $6 \mathrm{a}$ & $21 / 2$ & lag weiss & $2 \cdot 6$ & $33 \mathbf{a}$ & 5 & bl \\
\hline $1 \cdot 43$ & $15 \mathrm{~b}$ & $13 / 4$ & Korkstein m. schwarz. Pappe & $2 \cdot 61$ & $26 \mathrm{a}$ & $5^{3} / 4$ & weisses Tuch und (talas \\
\hline 1.45 & $11 \mathrm{~b}$ & $51 / 2$ & iegel & $2 \cdot 7$ & $20 \mathrm{~b}$ & $1 \%$ & \\
\hline $1 \cdot 5$ & $1 \mathrm{~b}$ & 2 & weiss & $2 \cdot 9$ & $40 a$ & 39 & crême Vorhang und Glas \\
\hline $1 \cdot 5$ & $4 a$ & 4 & ech & $3 \cdot 0$ & $24 \mathrm{~b}$ & 5 & weisses Tuch und Glas \\
\hline $1 \cdot 5$ & $42 \mathrm{a}$ & 4 & lech & $3 \cdot 0$ & $33 \mathrm{~b}$ & 5 & rothes Glas \\
\hline $1 \cdot 5$ & $41 \mathrm{~b}$ & 5 & crême Vor & $3 \cdot 1$ & $30 \mathrm{a}$ & $21 / 4$ & doppelter dunkler Woll- \\
\hline 1.58 & $27 \mathrm{a}$ & $71 / 4$ & dicker schwarzer Vorhang & & & & vorhang \\
\hline $1 \cdot 6$ & $35 \mathrm{a}$ & $71 / 2$ & weisser Vorha & $3 \cdot 3$ & $28 \mathrm{a}$ & $11 / 2$ & crême Vorhang und Glas \\
\hline $1 \cdot 6$ & $43 \mathrm{a}$ & $51 / 2$ & Dac & & $14 \mathrm{a}$ & 7 & Gla \\
\hline $1 \cdot 64$ & $9 \mathrm{~b}$ & 7 & Dachpappe $\mathrm{u}$. & $3 \cdot$ & $16 \mathrm{~b}$ & 1 & Korkstein u. \\
\hline $1 \cdot 64$ & $10 \mathrm{~b}$ & 7 & Dachpap & & $40 \mathrm{~b}$ & $33 / 4$ & durchbro \\
\hline $1 \cdot 66$ & $39 \mathrm{a}$ & 6 & Dachziegel & $3 \cdot$ & $20 \mathrm{a}$ & $11 / 2$ & \\
\hline $1 \cdot 7$ & $36 \mathrm{~b}$ & 4 & Dach & $3 \cdot 71$ & $32 \mathrm{~b}$ & $7 \%$ & Ornamentglas und Glas \\
\hline 1.7 & $13 \mathrm{~b}$ & $\overline{5}$ & weisser Vorhang und Glas & & $30 \mathrm{~b}$ & $2 \%$ & crême Vorhang und Glas \\
\hline $1 \cdot 71$ & $31 \mathrm{a}$ & 7 & & $3 \cdot 85$ & $21 a$ & $61 / 2$ & mattes Glas \\
\hline 1.73 & $32 \mathrm{a}$ & $71 / 2$ & desgl. & $4 \cdot 0$ & $5 \mathrm{~b}$ & $3 / 4$ & Blech und weisses Holz \\
\hline $1 \cdot 8$ & $43 \mathrm{~b}$ & $51 / 2$ & Holzschalung u. Dachpappe & $4 \cdot 0$ & $19 \mathrm{a}$ & $11 / 2$ & Schiefer \\
\hline $1 \cdot 8$ & $37 \mathrm{~b}$ & $23 / 9$ & Dachziegel & $4 \cdot 0$ & $38 \mathrm{~b}$ & 3 & 5 cm dickes Rohglas u. Glas \\
\hline $1 \cdot 8$ & $41 \mathrm{a}$ & 5 & doppelter schwarzer Woll. & 4 . & $13 a$ & 5 & glas \\
\hline & & & . & & $19 \mathrm{~b}$ & $11 / 2$ & \\
\hline $1 \cdot 8$ & $7 \mathrm{a}$ & $51 / 2$ & Dachziegel & $5 \cdot 0$ & $28 \mathrm{~b}$ & $11 / 2$ & Store mit Maschen \\
\hline $1 \cdot 85$ & $29 \mathrm{a}$ & $61 / 2$ & crême Vorhang und Glas & $5 \cdot 0$ & $38 \mathrm{a}$ & 3 & Fensterglas n. Ornamentglas \\
\hline $1 \cdot 86$ & $18 \mathrm{a}$ & 7 & weisser Vorhang und Glas & $5 \cdot 3$ & $5 \mathrm{a}$ & $3 / 4$ & Blech, darüb. schwarzes Hola \\
\hline $2 \cdot 0$ & $1 \mathbf{a}$ & 2 & Holzbelag schwarz & $6 \cdot 0$ & $34 \mathrm{~b}$ & $11 / 2$ & $5^{\mathrm{cm}}$ dickes Rohglas \\
\hline $2 \cdot 0$ & $39 \mathrm{~b}$ & 6 & Dachziegel & $6 \cdot 66$ & $34 \mathrm{a}$ & $1 \%$ & Ornamentglas \\
\hline $2 \cdot 0$ & $6 \mathrm{~b}$ & $|21 / 2|$ & & & & & \\
\hline
\end{tabular}

